\title{
GABAergic Synaptic Plasticity during a Developmentally Regulated Sleep-Like State in C. elegans
}

\author{
Nooreen S. Dabbish and David M. Raizen \\ Department of Neurology and Center for Sleep and Circadian Neurobiology, Perelman School of Medicine at the University of Pennsylvania, Philadelphia, \\ Pennsylvania 19014
}

Approximately one-fourth of the neurons in Caenorhabditis elegans adults are born during larval development, indicating tremendous plasticity in larval nervous system structure. Larval development shows cyclical expression of sleep-like quiescent behavior during lethargus periods, which occur at larval stage transitions. We studied plasticity at the neuromuscular junction during lethargus using the acetylcholinesterase inhibitor aldicarb. The rate of animal contraction when exposed to aldicarb is controlled by the balance between excitatory cholinergic and inhibitory GABAergic input on the muscle. During lethargus, there is an accelerated rate of contraction on aldicarb. Mutant analysis and optogenetic studies reveal that GABAergic synaptic transmission is reduced during lethargus. Worms in lethargus show partial resistance to $\mathrm{GABA}_{\mathrm{A}}$ receptor agonists, indicating that postsynaptic mechanisms contribute to lethargusdependent plasticity. Using genetic manipulations that separate the quiescent state from the developmental stage, we show that the synaptic plasticity is dependent on developmental time and not on the behavioral state of the animal. We propose that the synaptic plasticity regulated by a developmental clock in C. elegans is analogous to synaptic plasticity regulated by the circadian clock in other species.

\section{Introduction}

Nervous system remodeling is an ancient, widespread phenomenon seen in animals ranging from cnidarians to vertebrates (Nakanishi et al., 2008; Watanabe et al., 2009). During developmental transitions, holometabolous insects - those that undergo a complete metamorphosis-show profound remodeling (Tissot and Stocker, 2000). At the insect neuromuscular junction (NMJ), adult motoneurons (MNs) remodel from larval precursors (Levine and Truman, 1982). Both the muscle and the $\mathrm{MNs}$ reorganize at the NMJ, and this reorganization is dependent on signaling through steroid hormones (Hegstrom and Truman, 1996). In Drosophila, synapse elimination begins with postsynaptic dismantling of the NMJ (Liu et al., 2010b).

Remodeling occurs not only as a function of nervous system development, but also as a function of circadian clock and sleep/ wake cycles. In Drosophila, remodeling of pigment-dispersing factor-positive neurons is observed as a function of circadian

\footnotetext{
Received Feb. 10, 2011; revised Sept. 9, 2011; accepted Sept. 19, 2011.

Author contributions: N.S.D. and D.M.R. designed research; N.S.D. and D.M.R. performed research; N.S.D. and D.M.R. analyzed data; N.S.D. and D.M.R. wrote the paper.

D.M.R. is supported by R01 NS064030-01A1 and by a National Alliance for Research on Schizophrenia and Depression (NARSAD) Young Investigator Award. N.S.D. is supported by T32 HL07953. We are grateful to Jean-Louis Bessereau for the gift of anti-UNC-49 antibodies and for detailed staining protocols. We thank Erik Jorgensen, Cheryl Van Buskirk, and the C. elegans Genetics Center (which is funded by the NIH National Center for Research Resources) for providing strains and Jazz Pharmaceutical for providing sodium oxybate. We thank Annesia Lamb, Robert Driver and Kun He Lee for technical assistance. For comments on this manuscript, we thank Matt Nelson, Nick Trojanowski, Sarah McLoughlin, and Todd Lamitina.

The authors declare no competing financial interests.

Correspondence should be addressed to David M. Raizen Department of Neurology, Perelman School of Medicine at the University of Pennsylvania, 464 Stemmler, 415 Curie Boulevard, Philadelphia, PA 19014. E-mail: raizen@mail.med.upenn.edu.

DOI:10.1523/JNEUROSCI.0742-11.2011

Copyright $\odot 2011$ the authors $\quad 0270-6474 / 11 / 3115932-12 \$ 15.00 / 0$
}

time (Fernández et al., 2008). At the Drosophila NMJ, there are circadian changes in the size of MN terminals (Mehnert et al., 2007). Zebrafish display circadian changes in the number of presynaptic boutons in hypocretin neurons. In addition, there is a small effect of the sleep/wake behavioral state on synaptic plasticity in the hypocretin neuron terminals (Appelbaum et al., 2010). In Drosophila subjected to sleep deprivation, increases in levels of both presynaptic and postsynaptic proteins (Gilestro et al., 2009) and an increase in the number of synaptic terminals in clock neurons (Donlea et al., 2009) suggest that synaptic plasticity is also influenced by the animal's behavioral state (sleep vs wake).

To further explore the molecular and genetic underpinnings of synaptic change in a system that captures developmental, clock-like, and sleep/wake features, we chose to examine synaptic transmission during Caenorhabditis elegans larval development. Development from embryo to reproductive adulthood in C. elegans is punctuated by four molts, which separate the four larval stages from each other and the fourth larval stage from the adult stage. At the end of each larval stage, there is a stage called lethargus. Lethargus is a behaviorally quiescent period characterized by the sleep-like properties of reversibility, increased arousal threshold, and homeostasis (Raizen et al., 2008). In addition, lethargus timing is coupled to the expression of $\operatorname{lin}-42$, the $C$. elegans ortholog of the circadian protein PERIOD (Jeon et al., 1999). Therefore, larval development in C. elegans captures elements of both developmental clock-controlled changes and behavioral state-controlled changes.

To study the mechanisms of synaptic alterations during lethargus, we used pharmacological and optogenetic approaches to examine synaptic transmission at the NMJ. We show that synaptic transmission is altered during lethargus periods. We found 
that this alteration occurs during lethargus but not during other quiescent behavioral states. During lethargus, there is a reduction in transmission at GABAergic synapses at the body wall NMJ. We further show that this reduction can be explained in part by a change in the GABAergic synapse's postsynaptic function.

\section{Materials and Methods}

General methods and strains. Worms were cultured and handled as described previously (Brenner, 1974). All experiments were performed on hermaphrodites. Unless otherwise noted, worms were grown at $20^{\circ} \mathrm{C}$ on NGM agar plates supplemented with streptomycin sulfate at $50 \mu \mathrm{g} / \mathrm{ml}$ and fed the streptomycin-resistant Escherichia coli strain DA837 (Davis et al., 1995). C. elegans Variety Bristol strain N2 was used as the wild-type strain. Strains used were IB16 ceh-17(np1) I, JT1734 goa-1(sa734) I, CB156 unc-25(e156) III, CB407 unc-49(e407) III, CB382 unc-49(e382)III, FK234 egl-4(ks62) IV, DA521 egl4(ad450) IV, NM1968 slo-1( js379) V, CB6055 bus-8(e2698) X, MH1955 nhr-25(ku217) X, PS5009 pha-1(e2132ts) III;him-5(e1490) V;syEx723 [hs::LIN-3C;Pmyo2:GFP;pha-1(+)], EG5025 [Punc-47:ChR2::mCherry], and EG5096 [Punc-17:ChR2::mCherry].

The transgenes Punc-17:ChR2::mCherry and Punc-47:ChR2::mCherry were crossed into unc-25 and into goa- 1 mutants by isolating from heterozygotes progeny that showed the Unc- 25 and Goa-1 phenotypes, respectively, and also showed red fluorescence. Homozygosity of the transgene was then confirmed by looking for $100 \%$ progeny with red fluorescence.

RNA interference (RNAi) experiments to knock down $n h r-23$ were performed by the feeding method (Timmons and Fire, 1998) using bacteria expressing $n h r-23$ genomic fragment (Kamath et al., 2003). Wildtype worms were hatched in the absence of food and placed on an NGM agar surface fully seeded with a lawn of bacteria expressing $n h r-23$ double-stranded RNA and supplemented with $1 \mathrm{~mm}$ isopropyl- $\beta$-Dthiogalactopyranoside (IPTG). Effectiveness of the RNAi was evident based on the appearance of several larvae arrested in the third larval stage (L3) molt, a phenotype described previously for $n h r-23($ RNAi) (Kostrouchova et al., 2001; Frand et al., 2005). Animals that escaped this arrest were studied in the aldicarb assay during the L4 and L4 lethargus stages.

A Leica MS5 stereomicroscope was used to select worms for assays and for performing the aldicarb assays. For adult assays, L4 worms were selected the day before the assay and maintained at $20^{\circ} \mathrm{C}$ until the time of the experiment. For L4 assays, early L4 worms were selected on the basis of age, size, and vulval developmental stage. L4 lethargus animals were selected on the basis of age, size, morphological appearance of the uterus and vulva, and the absence of pharyngeal pumping for $10 \mathrm{~s}$.

Aldicarb assays. Aldicarb (Ultra Scientific; product no. PST-940) assays were performed on hermaphrodites as described previously (Mahoney et al., 2006). Because of day-to-day variability, comparisons were made only between worms assessed on the same day and on the same batch of aldicarb plates. To prepare agar plates containing $1 \mathrm{~mm}$ aldicarb, one part of $10 \mathrm{~mm}$ aldicarb stock solution was added to nine parts of molten NGM agar precooled to $50-60^{\circ} \mathrm{C}$. The $10 \mathrm{~mm}$ aldicarb stock solution was prepared by gently rocking the drug in water for $4-16 \mathrm{~h}$. Unless noted, aldicarb assays were performed on NGM plates that were not seeded with bacteria.

For the aldicarb sensitivity assay during the first two larval stages (see Fig. 1), worms were synchronized using an alkaline bleach preparation (Stiernagle, 2006). L1 larvae, developmentally arrested by hatching in a food-free M9 solution, were plated onto food at two time points $13 \mathrm{~h}$ apart to allow for a complete time course. Worms from each of these two cohorts of worms were transferred to aldicarb plates every $2 \mathrm{~h}$ and assessed for paralysis after $1 \mathrm{~h}$. Worms were considered to be paralyzed if they moved neither spontaneously nor in response to three prods on the head. Paralyzed worms were removed from the plate.

For L4 and adult time course to paralysis on aldicarb assays, worms were obtained from standard growing cultures, from synchronized (by alkaline bleach method) cultures, or by chunking starved plates. Worms were transferred to unseeded aldicarb plates with the aid of bacteria on a platinum wire tip. These worms were inspected every 15 min under $25 \times$ total magnification. Worms were considered to be paralyzed if they did not move in response to three anterior prods with a wire. Once the worms became less mobile they were aligned in the center of the plate to facilitate rapid observation of the worms at that time point.

Aldicarb sensitivity of satiety worms and their controls was assessed on aldicarb plates fully seeded with the bacteria HB101. The assessments of aldicarb sensitivity and satiety behavior were done blind to prior treatment of the worms (satiety-inducing conditions vs controls). Satiety behavior was induced by the starvation refeeding method as described previously (You et al., 2008). Satiated worms showed a delayed latency to resumption of movement and feeding $(20.8 \pm 5.5 \mathrm{~s})$ relative to control well fed worms $(4.6 \pm 4.7 \mathrm{~s})(p=0.0001)$.

The LIN-3 heat shock aldicarb assay was performed blinded to condition (with or without heat shock). Worms were heat shocked by immersing the agar plate housing them in a water bath set to $33^{\circ} \mathrm{C}$ for $30 \mathrm{~min}$. The aldicarb assay was started $2 \mathrm{~h}$ after the end of the heat shock. We found that the heat shock reproduced the behavioral effects (cessation of pumping and locomotion) reported for LIN-3 overexpression (Van Buskirk and Sternberg, 2007).

Pentylenetetrazole assay. Pentylenetetrazole (PTZ; Sigma catalog no. P6500) was included in the NGM at a concentration of $5 \mathrm{mg} / \mathrm{ml}$ as described previously (Locke et al., 2009). These plates were seeded with the bacteria DA837. Plates containing PTZ were stored overnight at $4^{\circ} \mathrm{C}$. Mid-L4, lethargus, and adult worms were plated on the PTZ plates prewarmed to room temperature and then observed at 15,30, and $60 \mathrm{~min}$. At each of these time points, worm behavior was categorized as follows: (1) normal crawling behavior; (2) posterior paralysis; (3) complete paralysis; and (4) convulsions (also referred to as "head bobs") (Williams et al., 2004; Locke et al., 2009). In this assay, paralyzed worms were neither prodded nor removed from the plate. The mid L4 and lethargus observations were performed blinded to stage. Because of their larger size, it was not possible to examine adult worms blinded to stage.

Muscimol and sodium oxybate assays. Muscimol (Sigma, catalog no. G019) and sodium oxybate (a gift from Jazz Pharmaceuticals) assays were performed as described previously (de la Cruz et al., 2003), except that a category 5 was added to designate complete flaccid paralysis. Specifically, the categories are as follows: category 0 , the worm moved away rapidly from the mechanical stimulus; category 1 , the worm briefly contracted and relaxed and then moved away from the stimulus; category 2, the worm contracted and then relaxed while at the same time making a small displacement away from the stimulus; category 3 , the worm contracted and then relaxed but showed no displacement; category 4, the worm contracted incompletely and then relaxed and showed no displacement; category 5 , the worm showed flaccid paralysis and did not respond at all to the mechanical stimulus. Assays were carried out on $0.5 \mathrm{~mm}$ muscimol and $10 \mathrm{mg} / \mathrm{ml}$ sodium oxybate plates.

Optogenetic experiments. All-trans retinal (ATR; Sigma, catalog no. 048K5004) plates were made as described previously (Liu et al., 2009), except that $48 \mu \mathrm{l}$ of $100 \mathrm{~mm}$ ATR stock was mixed with $3 \mathrm{ml}$ of DA837. Also, ATR plates were fully seeded with bacteria. An enriched population of L4 worms was obtained by placing gravid adult parents on ATR plates overnight. Parents were removed the next day, and L4s were selected from the plates $2 \mathrm{~d}$ later. ATR plates were kept in the dark until the time of the assay. L4 worms were transferred to individual, unseeded ATR plates for imaging on the base of a Leica MZ16F stereomicroscope equipped with a GFP filter. They were observed continuously with dim white light reflected onto the worm by a mirror in the microscope base. The worms were exposed to a blue light pulse for $\sim 30$ s using a manually controlled shutter. After obtaining images of the worm before and during the blue light pulse, body length was measured manually using LAS software (Leica), using an increase in the overall intensity of light to indicate when the shutter was open. Body length measurements were done blinded to condition (lethargus vs L4). Optogenetic experiments comparing L4 to L4 lethargus worms were done on the same day.

Immunostaining and image analysis. The anti-UNC-49 was a gift from the Bessereau Laboratory, Institut de Biologie de l’École Normale Supérieure, Paris, France (Gendrel et al., 2009), which generated the antibody by immunizing a rabbit with amino acids $23-67$ of the $\mathrm{N}$ terminus of UNC-49 fused to glutathione- $S$-transferase. The N terminus of UNC-49 is common to all isoforms of the protein (Bamber et al., 1999). We observed no staining in unc-49(e407) mutants, indicating that this anti- 
body is specific. The DYN-1 antibody (Hadwiger et al., 2010) was obtained from the Developmental Studies Hybridoma Bank at the University of Iowa, Iowa City, IA. Two-color coimmunostaining with these antibodies was done using the freeze-crack method as described previously (Leung et al., 1999). During a 30 min collection period, L4 lethargus and mid-L4 worms were picked into M9 buffer precooled on ice. The worms were transferred to a glass slide and, after placing a coverslip on top of the worms, excess liquid was removed with a Kimwipe (Kimberly Clark) under stereomicroscopical observation until many worms were observed to explode. After a $1-2 \mathrm{~h}$ incubation on dry ice, the worms were fixed for $15 \mathrm{~min}$ with methanol at $20^{\circ} \mathrm{C}$, rinsed with PBSTriton X-100, blocked in 1\% BSA, and then incubated overnight simultaneously in both the UNC-49 and DYN-1 primary antibodies at 1:500 and 1:100 dilutions, respectively, at room temperature. The next day, the worms were rinsed with PBS- Triton X-100 and then incubated for 4 hat room temperature in both $\mathrm{Cy} 3$-conjugated donkey-anti-rabbit secondary antibody (Jackson ImmunoResearch Laboratories, catalog no. 711165-152) at 1:800 dilution and in FITC-conjugated donkey-anti-mouse second antibody (Jackson ImmunoResearch Laboratories, catalog no. 715-095-150) at 1:400 dilution. Following two PBS rinses, the worms were mounted in Immunogold (Invitrogen, catalog no. P36930). Slides were allowed to cure overnight in the dark at room temperature before sealing with nail polish.

Images were obtained with a 1.4 numerical aperture $100 \times$ objective on a Leica DM5500 epifluorescent microscope and analyzed using Leica LAS software. In brief, under the "quantify" function in LAS a line was drawn through several fluorescent puncta. LAS software quantified the signal intensity along the line, and the peak intensity values along the line were recorded and averaged for each animal. The number of puncta measured per animal ranged from 10 to 11 . The background fluorescence of each worm, which was obtained by measuring the average pixel intensity in a $10-30$ pixel $^{2}$ rectangular area in the animal's mid-body away from the synaptic puncta, was subtracted from the average synaptic puncta peak intensity. For each worm, the background-corrected peak intensity for UNC-49 staining was divided by the background-corrected peak intensity for DYN-1 staining. We report in Results the average \pm SEM of this ratio for eight worms in the lethargus group and nine worms in the mid-L4 stage.

Quantitative reverse-transcriptase PCR. A synchronized population of worms was plated at a density of 200 worms per NGM plate seeded with a lawn of DA837 bacteria. L4 or L4 lethargus worms were collected at 40 worms per sample into $100 \mu \mathrm{l}$ of TRIzol (Invitrogen, catalog no. 15596$026)$ and flash frozen. RNA isolation was performed using RNeasy MinElute Columns (Qiagen, catalog no. 74204). Ten microliters of sample was made into cDNA by reverse strand synthesis (RT-PCR) (Roche First Strand cDNA Synthesis, catalog no. 11483188001). Quantitative PCR was performed using the SYBR Green Master Mix (Applied Biosystems, catalog no. 4309155) using an ABI Thermocycler 7500. Amplification was performed using oligonucleotides for unc-49A, unc-49B, and unc49C designed using Primer3 (http://frodo.wi.mit.edu/primer3/) and the reference gene $p m p-3$. We chose to use $p m p-3$ as the reference gene because previous work has established that it shows one of the most stable expressions across experiments among C. elegans genes (Hoogewijs et al., 2008). Indeed, we observed no change in expression of pmp-3 during larval development (data not shown). We verified that all amplification products migrated at the correct predicted size after electrophoretic separation in an agarose gel. The sequence of primers used is available upon request. The normalized expression of the unc- 49 isoforms in L4 lethargus relative to mid L4 stage was determined by calculating $2^{-\Delta \Delta \mathrm{Ct}}$ (Livak and Schmittgen, 2001).

Statistical analysis. The time course to paralysis on aldicarb was analyzed using the log rank sum test (Bland and Altman, 2004). Categorical data such as that obtained from observing the behavioral response to PTZ and to muscimol was analyzed using Fisher's exact test. Parametric data that were normally distributed such as that obtained in optogenetic experiments were analyzed with a two-sample, two-tailed Student's $t$ test. Parametric data that were clearly not normally distributed normally, such as those presented in Figure $1 E$, were analyzed using a Mann-
Whitney $U$ test (http://elegans.swmed.edu/ leon/stats/utest.html). Significance was set to 0.05 .

\section{Results}

\section{L1 lethargus and L4 lethargus worms are aldicarb hypersensitive}

The C. elegans NMJ is a well characterized synapse (Wang et al., 2001; Richmond, 2005; Seifert et al., 2006) suited for the study of alterations in synaptic function during lethargus. C. elegans body muscle receives input from excitatory cholinergic motor neurons and inhibitory GABAergic neurons. The balance of these two inputs regulates the degree of muscle contraction (Fig. $1 A$ ).

A powerful method for assessing alterations in synaptic transmission at the NMJ is to use the drug aldicarb (Mahoney et al., 2006). Aldicarb inhibits the enzyme acetylcholinesterase, causing the accumulation of acetylcholine (ACh). This leads to increased excitatory input and eventually to hypercontractive paralysis. The rate at which worms become paralyzed reflects the balance of excitatory cholinergic and inhibitory GABAergic inputs onto the muscle (Vashlishan et al., 2008) (Fig. 1A).

Prior dose-response experiments with aldicarb have shown that $1 \mathrm{~mm}$ aldicarb in wild-type adult worms at $5 \mathrm{~h}$ of treatment is saturating. That is, concentrations greater than $1 \mathrm{~mm}$ do not result in further increases in overall paralysis (Nonet et al., 1997). Although during lethargus worms display reduced locomotion and reduced responsiveness, they remain responsive to harsh touch (Raizen et al., 2008) and, when placed on aldicarb, are in fact hyperactive until paralysis sets in. Therefore, it is possible to test the effect of aldicarb on lethargus worms. In keeping with the published protocol, we considered worms to be paralyzed if they did not move at all in response to three consecutive prods to the anterior body with a platinum wire (Mahoney et al., 2006).

To measure aldicarb sensitivity as a function of developmental time, we hatched eggs in buffer without food such that the first larval stage animals, L1s, were developmentally arrested and synchronized (Stiernagle, 2006). We tested the L1s on aldicarb as a function of time after first exposure to food, which resumed their development. Before lethargus, over $99 \%$ of L1s were moving after $1 \mathrm{~h}$ on $1 \mathrm{~mm}$ aldicarb (Fig. $1 B$ ). Lethargus worms were identified based on an absence of pharyngeal pumping at a developmental age of $16 \mathrm{~h}$. In contrast to earlier time points, only $4.1 \pm$ $1.1 \%$ of worms were moving after exposure to aldicarb from the $16 \mathrm{~h}$ to the $17 \mathrm{~h}$ time point (Fig. $1 \mathrm{~B}$ ), indicating that most of the worms were paralyzed. Worms exposed to aldicarb from 18 to $19 \mathrm{~h}$ of age displayed an intermediate value of $42.2 \pm 13.2 \%$ moving worms. Importantly, worms were also tested at $21 \mathrm{~h}$ after exposure to food, which corresponds to the beginning of the second larval stage (L2). At this time point, aldicarb sensitivity returned to baseline with $99.2 \pm 1.3 \%$ of worms moving. In summary, nearly all L1 and L2 worms were moving in this assay with the exception of the sharp hypersensitivity observed in L1 lethargus worms.

To test whether the L1 lethargus aldicarb hypersensitivity extends to other lethargus periods, we measured aldicarb sensitivity in the lethargus period that occurs at the end of the fourth larval stage, L4 lethargus. We compared aldicarb sensitivity of early L4 worms to that of L4 lethargus worms by assessing paralysis every $15 \mathrm{~min}$. The rate of paralysis on aldicarb was much faster during L4 lethargus than in early L4 worms (Fig. $1 C$ ). The point at which half the L4 lethargus worms were paralyzed was $\sim 2 \mathrm{~h}$ earlier than the point at which half of the early L4 worms were paralyzed. We observed a similar hypersensitivity to aldicarb when we compared L4 lethargus animals to adult animals (Fig. 1D). 

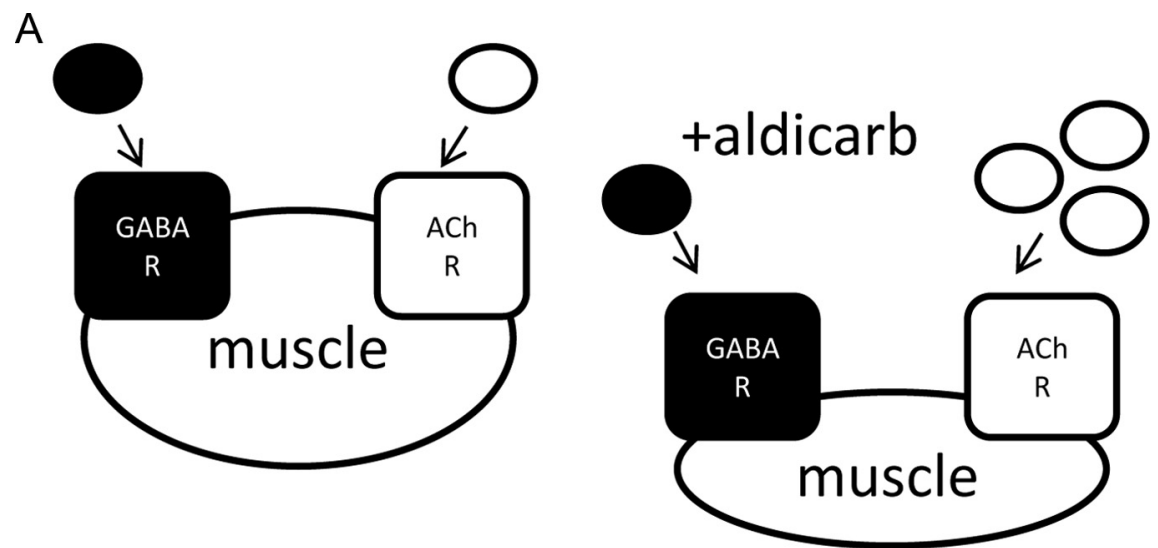

B
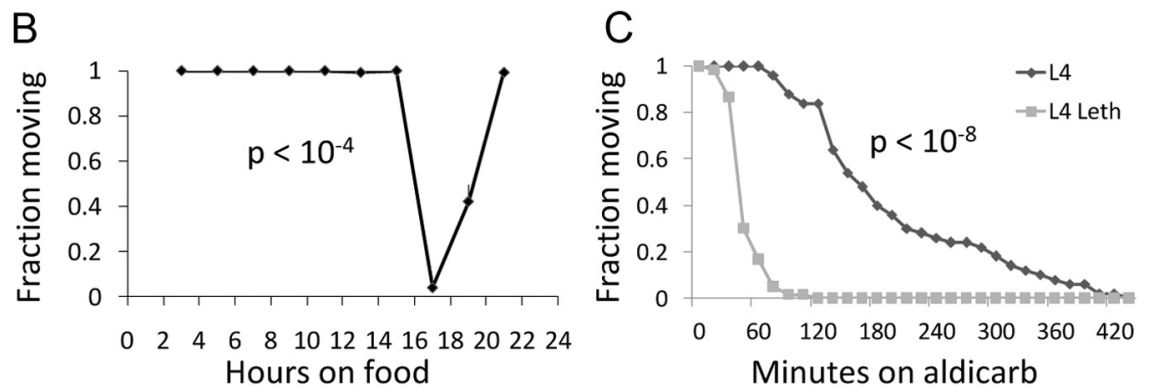

D

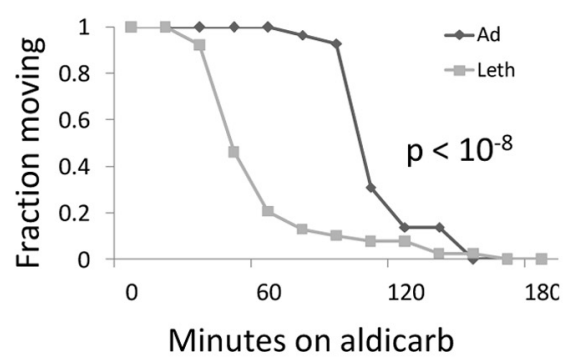

that the mechanism of paralysis in the aldicarb assay consists of muscle hypercontraction, as proposed previously (Mahoney et al., 2006).

Developmental time and not behavioral state confers aldicarb hypersensitivity The aldicarb hypersensitivity could be explained either by the developmental stage of the animal or by the behavioral state of the animal, which is different during lethargus. The distinction between developmental time and behavioral state in $C$. elegans is analogous to the distinction between circadian time and behavioral state (sleep versus wake) in mammals. The fact that lethargus animals placed on aldicarb show hyperactivity before paralysis suggested to us that the accelerated paralysis is not a result of the sleep-like behavioral state, but rather is a result of the developmental stage of the animal. We performed several additional experiments to further distinguish between the effects of developmental time and the sleep-like behavioral state of the animals.

Two adult quiescent behaviors resembling lethargus behavior have been described; both are marked by decreased locomotion and cessation of pharyngeal pumping, and both are regulated by the gene egl-4, which also regulates lethargus. The first behavior occurs under conditions of satiety. Worms become quiescent when fed a highly preferred bacteria or when refed bacteria after a fast (You et al., 2008). We induced satiety behavior by the fasting-refeeding method as described previously (You et al., 2008). Successful induction of this satiated state was evident by the observation of cessation of locomotion and pumping (see Materials and Methods). Satiated worms, however, did not display significant hypersensitivity to aldicarb (Fig. 2A).

A second recently described quiescent behavior follows induced overexpression of the gene lin-3, which encodes an epidermal growth factor (Van Buskirk and

To test the notion that the immobility on aldicarb during L4 lethargus is a result of accelerated contraction (Fig. $1 \mathrm{~A}$ ) and not a consequence of a reduced propensity to move, we measured the rate of body muscle contraction as a function of time on the drug. Muscle contraction is reflected by shortening of the body, which can be readily measured. We found that the rate of body contraction during the L4 lethargus stage was significantly faster than the rate of body contraction during the mid L4 stage (Fig. $1 E$ ). Interestingly, worms in the L4 stage as well as in the L4 lethargus stage showed a U-shaped pattern of body shortening on aldicarb, with an initial contraction followed by a partial relaxation (Fig. 1E). This relaxation may be explained by desensitization of cholinergic receptors (Boulin et al., 2008) or by muscle fatigue but occurs later than the stage at which paralysis sets in. This suggests
Sternberg, 2007). Two hours after heat shock, we observed the quiescent behavior described previously (data not shown). We observed no increase in aldicarb sensitivity after heat shock induction of the LIN-3C gene in comparison to control animals of the same genotype that were not heat shocked (Fig. 2B). Rather, heat shock treatment itself caused slight aldicarb resistance in wild-type animals and in animals carrying the inducible LIN-3C transgene (Fig. $2 \mathrm{~B}$ ). Therefore, the lethargus-like phenotypes induced by LIN-3C overexpression do not include aldicarb hypersensitivity.

We next examined the aldicarb sensitivity of mutants that are known to cause reduced quiescence during lethargus and lighter sleep-like behavior as evidenced by increased responsiveness. We examined the behavior of animals with loss of function muta- 

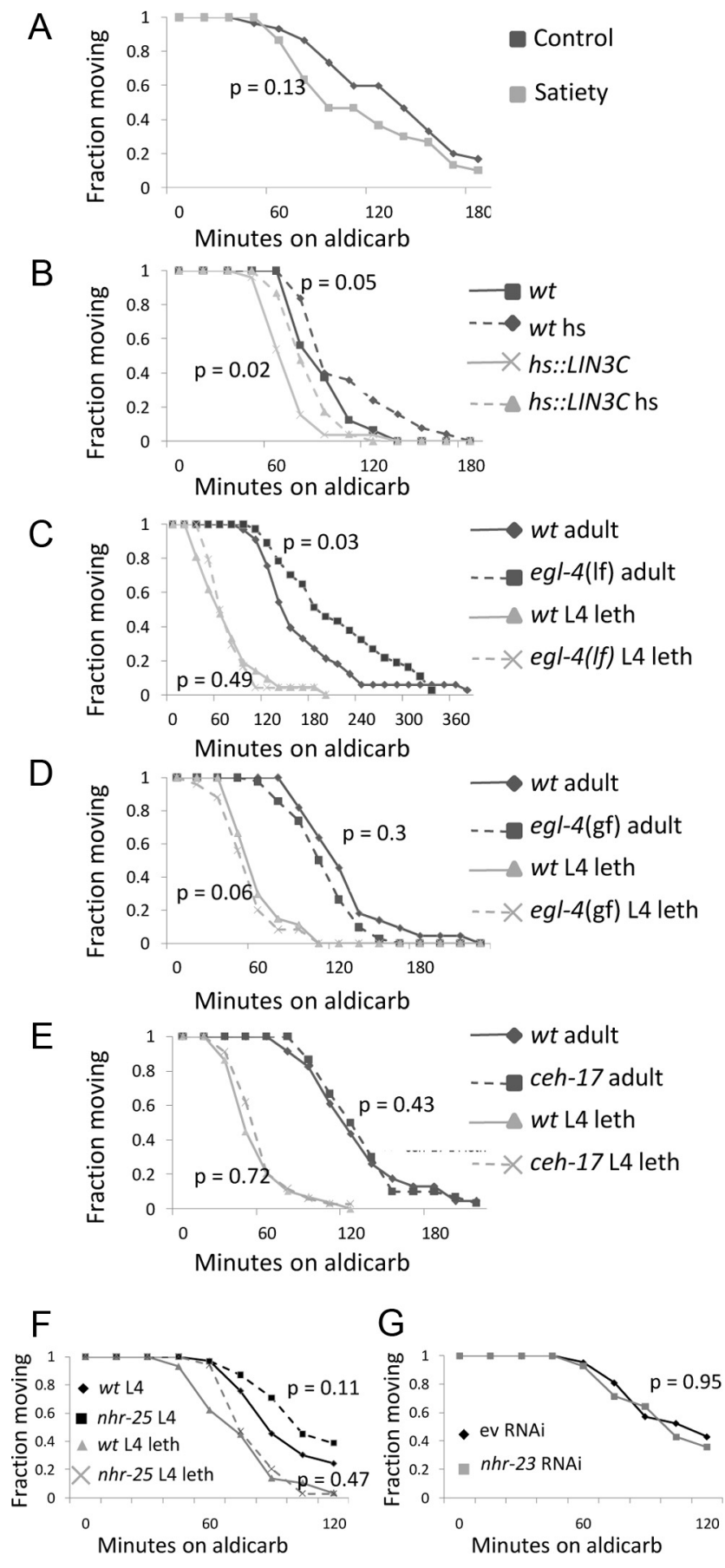

Figure 2. Developmental time and not behavioral state confers aldicarb hypersensitivity. $A$, Satiated worms (gray line) do not display significant acceleration relative to control worms (black line) in the time course to paralysis on aldicarb. $\boldsymbol{B}$, Heat shocked wild-type animals (dashed black line, $n=25$ ) displayed aldicarb-resistance relative to non-heat-shocked controls (solid black line, $n=16$ ), as did the heat-shocked strain carrying an extrachromosomal array of hs:: LIN3C (dashed gray line, $n=23$ ) in comparison to non-heat shocked controls of the same strain (solid gray line, $n=26$ ). C, During the adult stage, egl-4 loss-of-function (If) mutants (dashed black line, $n=37$ ) are aldicarb-resistant relative to adult wild-type $(w t)$ worms (solid black line, $n=33$ ). During the $L 4$ lethargus (leth) stage, egl-4(If) (dashed gray line, $n=24$ ) are not different from wild-type worms (solid gray line, $n=21$ ). $D$, During the adult stage, the aldicarb sensitivity of egl-4 gain-of-function ( $\mathrm{gf}$ ) mutants (dashed black line, $n=25$ ) is not different from that of adult wild-type worms (solid black line, $n=27$ ). During the $L 4$ lethargus stage, egl-4(gf) (dashed gray line, $n=42$ ) mutants are not different from wild-type worms (solid gray line, $n=22$ ). $\boldsymbol{E}$, During the adult stage, ceh-17 mutants (dashed black line, $n=30$ ) show no difference from wild-type worms (solid black line, $n=23$ ). During $L 4$ lethargus, ceh-17 mutants (dashed gray line, $n=24$ ) show no difference from wild-type worms (solid tions in egl-4, a gene required for the normal expression of sleeplike behavior during lethargus (Raizen et al., 2008). As reported previously (Daniels et al., 2000), we found that egl-4 adults are aldicarb resistant (Fig. 2C). In contrast to the adult result, egl-4 loss of function mutants in L4 lethargus showed a wild-type time course to paralysis on aldicarb (Fig. 2C). A gain-of-function egl-4 mutant, ad450sd, in which sleep-like behavior is observed during the adult stage outside of lethargus, showed a small but not significant aldicarb hypersensitivity both in adults and in L4 lethargus (Fig. 2D). Therefore, although egl-4 mutations affect sleeplike behavior, they do not affect the aldicarb hypersensitivity during lethargus.

Finally, we tested animals that were mutant for the gene ceh17. ceh-17, which encodes a paired-like homeodomain transcription factor, is required for the development and function of the interneuron ALA (Van Buskirk and Sternberg, 2007, 2010). ALA is required for the full expression of $C$. elegans sleep-like behavior during lethargus. Animals lacking ALA function are more active during lethargus (Van Buskirk and Sternberg, 2007), and ceh-17 mutants show increased responsiveness during lethargus ( $R$. Driver and D. Raizen, unpublished data). In the aldicarb sensitivity assay, however, ceh-17 mutant adults and L4 lethargus animals showed a wild-type time course to paralysis (Fig. 2E).

Together, our results indicate that aldicarb hypersensitivity is conferred by the developmental stage (i.e., lethargus) of the animal and not by its behavioral sleep-like state. Previous work has identified the nuclear hormone receptors $n h r-23$ and $n h r-25$ as regulators of the molt (Gissendanner and Sluder, 2000; Kostrouchova et al., 2001; Frand et al., 2005), which is completed during lethargus. We therefore tested the aldicarb sensitivity during lethargus of $n h r-25$ ( $k u 217$ ) worms at this mutant's restrictive temperature as well as that of animals in which the gene $n h r-23$ was knocked down by RNAi method. We observed no change in aldicarb hypersensitivity phenotype during lethargus (Fig. $2 F, G)$. These results indicate that the developmental regulation of aldicarb hypersensitivity can be separated from the regulation of the molt.

We considered the possibility that, since animals during lethargus do not feed, their nutritional status may be compromised, and that starvation may effect changes in NMJ transmission. To address this possibility, we assessed the aldicarb sensitivity of adult worms $2 \mathrm{~h}$ after removal from their food source. We observed no change in the aldicarb sensitivity in these fasted animals (data not shown). We further found that addition of serotonin, a neurotransmitter that is released during feeding in C. elegans (Chase and Koelle, 2007), did not restore normal sensitivity to aldicarb during lethargus (data not shown). Therefore, we conclude that the effects of lethargus on aldicarb sensitivity are not explained by the absence of feeding.

\section{Aldicarb hypersensitivity can be partially explained by reduced GABAergic neuromuscular transmission}

The shorter time course to reach a hypercontracted state could be due either to increased cholinergic synaptic transmission (a

$\leftarrow$

gray line, $n=29) . \boldsymbol{F}, n h r-25(k u 217)$ mutants (dashed lines) were not different from wild-type worms (solid lines) during lethargus (gray lines, $n=29-34$ ) or during the mid L4 stage (black lines, $n=31-33)$. $\mathbf{G}$, The time course to paralysis on aldicarb during L4 lethargus of $n h r-$ 23/(RNAi)-treated animals ( $g r a y, n=14$ ) is not different from the time course of empty vector (ev) RNAi-treated animals (black, $n=21$ ). 
A

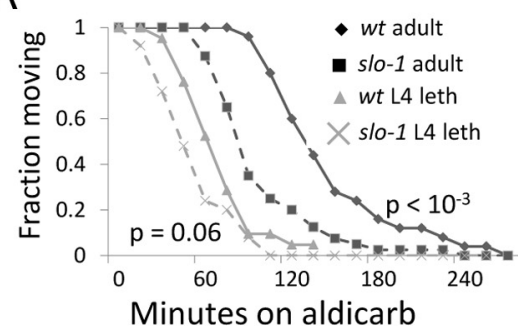

C

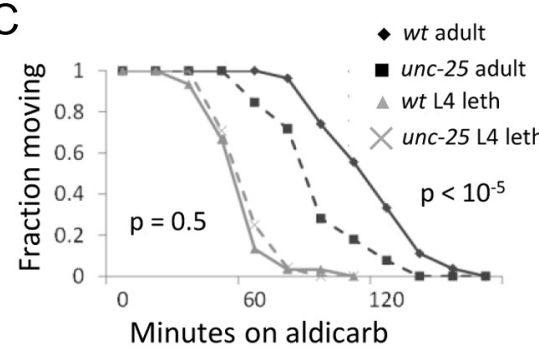

E

GABA MN stimulated

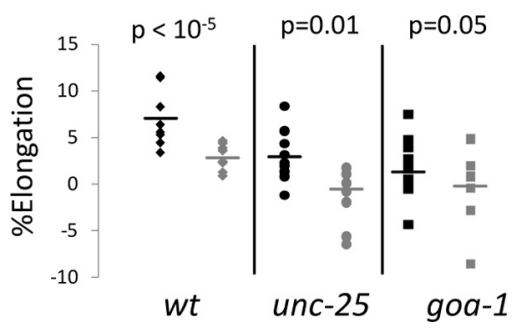

B
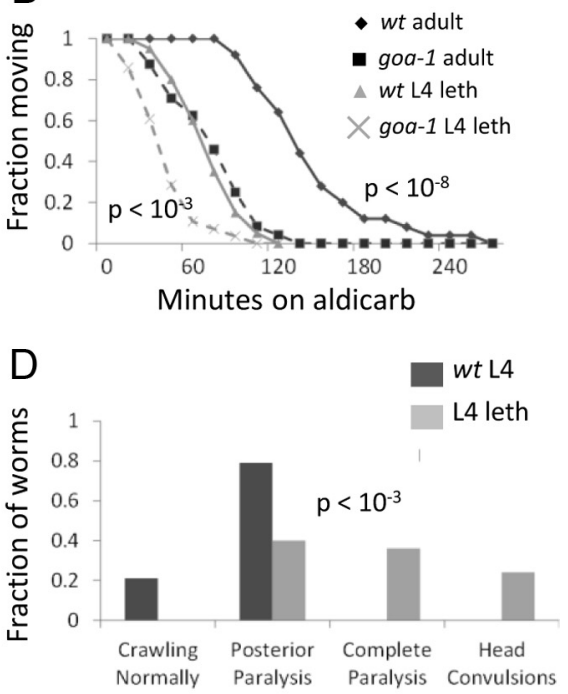

$\mathrm{F}$

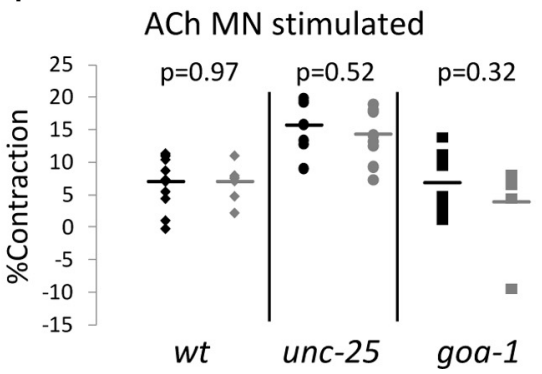

Figure 3. Decreased GABAergic synaptic transmission during lethargus. A, slo-1 mutant worms (dashed lines) show a significantly accelerated time course to paralysis in comparison to wild-type (wt) worms (solid lines) during the adult stage (black lines) but the acceleration during the L 4 lethargus (leth) stage (gray lines) does not reach statistical significance; $N=21-40 . B$, goa-1 mutants (dashed lines) show an accelerated time course to paralysis in comparison to wild-type worms (solid lines) as adults (black lines) and as $L 4$ lethargus worms (gray lines); $N=20-28$. C, During the adult stage, unc-25 mutants (dashed black line, $n=50$ ) have an accelerated rate of paralysis in comparison to wild-type worms (solid black line, $n=27$ ). During the L4 lethargus stage, the time course to paralysis in unc-25 (dashed gray line, $n=24$ ) is not different from that of wild-type worms (solid gray line, $n=30$ ). $D, L 4$ lethargus worms show a greater sensitivity to the GABA antagonist PTZ than mid-L4 worms. Shown is the fraction of worms

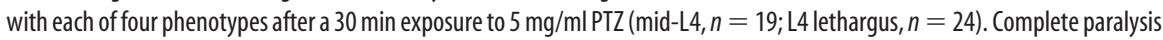
is not observed in mid-L4s at this 30 min time point. Convulsive head movements (head bobs) are not observed in mid-L4 at this time point, nor are they observed at any time point (Table 1). $\boldsymbol{E}$, L4 worms (black) are compared to $L 4$ lethargus worms (gray) in their response to optogenetic stimulation of GABAergic motor neurons. Horizontal bars denote mean responses. $F$, L4 worms (black) are compared to L4 lethargus worms (gray) in their response to optogenetic stimulation of cholinergic motor neurons. Horizontal bars denote mean responses.

higher drive to contract, causing hypercontraction earlier) or to decreased GABAergic synaptic transmission (a lower drive toward relaxation, allowing hypercontraction earlier) (Fig. $1 \mathrm{~A}$ ). To distinguish between these two possibilities, we studied mutants with well characterized defects in neuromuscular transmission. To test whether increased cholinergic synaptic transmission contributes to aldicarb hypersensitivity during lethargus, we studied mutants with increased cholinergic transmission. slo-1(js379) mutants have an early stop codon in a gene coding for the SLO-1 voltage- and calcium-sensitive potassium channel. slo-1(js379) mutants have prolonged synaptic release (Wang et al., 2001) and are hypersensitive to aldicarb (Wang et al., 2001). goa-1(sa734) mutants have an early stop codon in a gene coding for the C. elegans $\mathrm{G}_{\mathrm{o}} \alpha$ subunit (Robatzek et al., 2001) and are hypersensitive to aldicarb (Miller et al., 1999a). For both slo-1 and goa-1 mutant adults, the hypersensitivity to aldicarb is at least partially explained by increased cholinergic synaptic transmission (Miller et al., 1999b; Wang et al., 2001). If elevated cholinergic transmission was the sole explanation for the aldicarb hypersensitivity observed during lethargus, then these mutants should behave similarly to wild-type worms in the lethargus aldicarb assay. goa-1 mutants showed an accelerated time course to paralysis in comparison to wild-type worms, both in the adult stage as reported previously and during lethargus (Fig. 3B). The accelerated time course to paralysis in these mutants indicates that the rate of paralysis during lethargus observed in wild-type worms is not the maximum possible rate and that further acceleration is possible. This result further suggests that increased cholinergic transmission does not fully explain the aldicarb hypersensitivity during lethargus in wildtype worms. However, the possibility remains that increased cholinergic transmission does partially explain aldicarb hypersensitivity during lethargus, but that during lethargus these particular mutants have further increased cholinergic tone in comparison to wild-type worms. Indeed, slo-1 mutants, which during the adult stage are hypersensitive to aldicarb (Wang et al., 2001) (Fig. 3A), during lethargus showed only a small degree of hypersensitivity that did not reach statistical significance ( $p=$ 0.06; Fig. $3 A$ ). Therefore, GABA transmission mutants were used to further clarify the mechanism of aldicarb hypersensitivity during lethargus.

We used GABA-deficient mutants to test the idea that reduced GABAergic synaptic transmission contributes to aldicarb hypersensitivity during lethargus. The unc-25 gene encodes glutamic acid decarboxylase, an enzyme required for GABA synthesis, and unc-25(e156) mutant worms lack GABAergic neurotransmission (McIntire et al., 1993; Jin et al., 1999). If eliminating GABAergic transmission genetically in the lethargus aldicarb assay results in a wild-type time course to paralysis, this would suggest that reduced GABAergic synaptic transmission contributes to aldicarb hypersensitivity during lethargus. As reported previously (Vashlishan et al., 2008; Locke et al., 2009), unc-25 worms were hypersensitive to aldicarb as adults (Fig. 3C). In contrast, during L4 lethargus, unc-25 mutants demonstrated a wild-type time course to paralysis (Fig. $3 C$ ). This result suggests that there is reduced GABAergic synaptic transmission during lethargus, such that a further removal of GABA in unc-25 worms does not lead to further aldicarb hypersensitivity. Together with the goa-1 and slo- 1 results described above, the $u n c-25$ results point to reduced synaptic transmission from GABAergic neurons as the main mechanism explaining aldicarb hypersensitivity during lethargus.

To test for additional evidence of reduced GABAergic neuromuscular transmission, we used the drug pentylenetetrazole. In mammals, PTZ has been characterized as an antagonist to the mammalian $\mathrm{GABA}_{\mathrm{A}}$ receptor and can cause convulsions (Rocha et al., 1996). It can also cause convulsive body movements termed 
Table 1. L4 lethargus worms show increased susceptibility to the convulsant PTZ

\begin{tabular}{llrrr}
\hline Genotype & Time on $5 \mathrm{mg} / \mathrm{ml} \mathrm{PTZ}$ & \multicolumn{1}{l}{ Mid-L4 } & \multicolumn{1}{l}{ L4 Lethargus } & \multicolumn{1}{l}{ Adults } \\
\hline Wild-type & $15 \mathrm{~min}$ & $0 \%(19)$ & $32 \%(24)$ & \multicolumn{1}{l}{ ND } \\
& $30 \mathrm{~min}$ & $0 \%(19)$ & $24 \%(24)$ & $0 \%(31)$ \\
& $60 \mathrm{~min}$ & $0 \%(19)$ & $20 \%(24)$ & $0 \%(31)$ \\
unc-49(e407) & $30 \mathrm{~min}$ & $100 \%(18)$ & $93.1 \%(29)$ & $71.4 \%(28)$ \\
\hline
\end{tabular}

Shown are the percentages of animals with convulsive head movements at each of three time points after initial drug exposure. Number of animals tested is shown in parentheses. ND denotes not done.

"head bobs" in C. elegans mutants with reduced GABAergic synaptic transmission (Williams et al., 2004). Therefore, a convulsive response to PTZ can suggest a defect in GABAergic transmission (Williams et al., 2004).

Adult wild-type worms move normally when incubated on PTZ and display neither paralysis nor convulsive head bobs on doses up to $20 \mathrm{mg} / \mathrm{ml}$ (Williams et al., 2004). Mid-L4 animals respond to PTZ with phenotypes that include partial paralysis, but like adult animals they never displayed convulsive head movements (Table 1). In contrast to both adult and mid-L4 animals, L4 lethargus worms displayed convulsive head movements in addition to posterior body paralysis and complete paralysis (Fig. 3D and Table 1). The fact that some L4 lethargus animals show convulsions in response to PTZ, a behavior never observed in adult or L4 animals (see Table 1), supports the hypothesis that there is a reduction in GABAergic neuromuscular transmission during lethargus. Adult satiety worms were also tested on PTZ and no convulsions were seen (data not shown). This is consistent with the idea that the reduced GABAergic synaptic transmission is lethargus stage specific and not behavioral state specific.

An alternative explanation for the increased susceptibility to both aldicarb and PTZ during lethargus is that worms in lethargus are generally more sensitive to drugs due to enhanced cuticle permeability. Cuticle structure is dynamic during lethargus, as worms are secreting and assembling a new cuticle and escaping from the old cuticle of the prior larval stage. Others have used increased uptake of the fluorescent dye Hoechst as evidence of increased cuticle permeability (Moribe et al., 2004; Meli et al., 2010). Although we did not observe enhanced Hoechst uptake during lethargus (data not shown), bus- 8 mutants, which are known to have a more permeable cuticle (Partridge et al., 2008), show an accelerated rate of paralysis as adults (N. Dabbish and D. Raizen, unpublished observations). Therefore, to evaluate GABAergic signaling with a method that would not be affected by the degree of cuticle permeability, we studied neuromuscular transmission optogenetically.

Channelrhodopsin-2 (ChR2) is a cation channel that produces light-evoked electrical and behavioral responses when expressed in C. elegans muscle or neurons (Nagel et al., 2005). Neuromuscular transmission has been shown to be particularly amenable to optogenetic study. The effect of optogenetic stimulation of cholinergic motor neurons is evidenced by increased muscle contraction and therefore a shortening of the animal's posture (Liewald et al., 2008). Stimulation of GABAergic motor neurons, in contrast, causes muscle relaxation and therefore lengthening of the animal's posture. We observed these previously reported behavioral consequences to blue light (wavelength $450-490)$ stimulation of GABAergic and cholinergic neurons in adult animals (data not shown).

Using worms with ChR2 expressed in GABAergic neurons or in cholinergic motor neurons, we compared body length changes in response to optogenetic stimulation between early L4 and L4 lethargus worms. We studied wild-type worms as well as unc-25 and goa-1 mutants optogenetically. Optogenetic stimulation of GABAergic and cholingeric neurons in wild-type worms during the L4 stage gave the expected elongation and contraction phenotypes, respectively (Fig. $3 E, F$ ). During the L4 lethargus stage, wild-type worms contracted upon cholinergic stimulation to a similar degree seen in early L4 worms (Fig. 3F). In contrast, GABAergic stimulation during lethargus caused minimal elongation at $2.7 \pm 0.5 \%$ (Fig. $3 E$ ), far less than that seen during early L4 stage $\left(7.0 \pm 1.1 \%, p<10^{-5}\right)$. These optogenetic experiments provide nonpharmacological evidence for a reduction in synaptic transmission from GABAergic neurons during C. elegans lethargus.

Surprisingly, unc-25(e156) mutants, which are believed to lack GABA (McIntire et al., 1993), showed a small elongation $(2.9 \pm 1.0 \%)$ in response to GABAergic optogenetic stimulation during the L4 stage outside of lethargus (Fig. 3E). This result might be explained by unc-25(e156) mutants containing a small amount of residual GABA or by the use of neurotransmitters other than GABA to cause muscle relaxation. For example, there are neuropeptides reported to be expressed in GABAergic motor neurons (Kim and Li, 2004). Whatever the mechanism of residual relaxation in $u n c$ - 25 mutants, GABAergic optogenetic stimulation during lethargus resulted in no response (Fig. 3E), suggesting that there is complete lack of neurotransmission by GABAergic motor neurons.

Consistent with the literature reports of high cholinergic tone in goa-1 mutants, these mutants showed only small responses to stimulation of GABAergic or of cholinergic motor neurons (Fig. $3 E, F)$. Because cholinergic transmission is already very high in these mutants, stimulation of the cholinergic neurons optogenetically has only minimal effects. Conversely, stimulation of the GABAergic neurons optogenetically cannot overcome the high excitatory tone in these mutants and therefore results in minimal elongation $(3.1 \pm 1.0 \%)$, even outside lethargus.

\section{Worms in $\mathrm{L} 4$ lethargus are resistant to $\mathrm{GABA}_{\mathrm{A}}$ agonists}

Reduced GABAergic neuromuscular transmission can be explained by either reduced release of GABA by motor neurons and/or by reduced response of the muscle to GABA. To test for this second possibility, we assessed the worms' response to $\mathrm{GABA}_{\mathrm{A}}$ agonists. Worms placed on agar containing $\mathrm{GABA}_{\mathrm{A}}$ agonists demonstrate a range of responses reflecting the degree of relaxation of the body muscle (de la Cruz et al., 2003). The strongest response is complete flaccid paralysis, even in response to prodding. This is followed by the "rubber band" phenotype where, in response to prodding, the animals contract and relax without displacement. If reduced GABAergic signaling in lethargus were caused strictly by a presynaptic alteration, no difference in the severity of the rubber band response during lethargus would be expected following incubation on exogenous $\mathrm{GABA}_{\mathrm{A}}$ agonists. If, on the other hand, reduced GABAergic signaling in lethargus worms were caused partially by a postsynaptic alteration, a lower sensitivity to the exogenous GABA agonists would be expected.

Using a severity scale from 0 to 5 , worm responses to harsh anterior touch were scored after a $30 \mathrm{~min}$ exposure to $0.5 \mathrm{mM}$ muscimol (Fig. 4A). Relative to early L4 worms, worms in lethargus had a response shifted toward less severe responses, reflecting partial resistance to $\mathrm{GABA}_{\mathrm{A}}$ agonists and consistent with the postsynaptic alteration postulated above. Surprisingly, we found that $u n c-49$ mutants retained some response to the flaccid paralysis and rubber band-inducing effects of muscimol (Fig. 4B). This suggests that the effects of muscimol on posture and loco- 
A

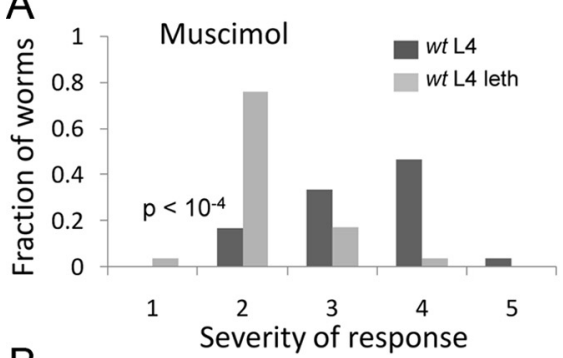

$\mathrm{B}$
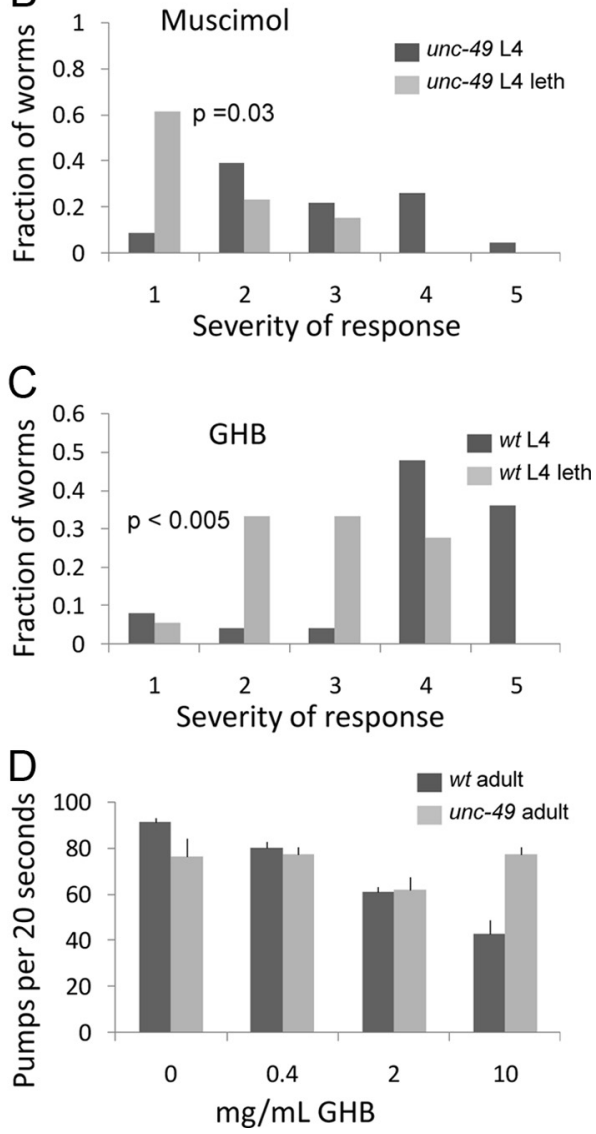

Figure 4. Worms in $L 4$ lethargus are less sensitive to $G A B A_{A}$ receptor agonists. $\boldsymbol{A}-\boldsymbol{C}$, Shown is the fraction of worms that displayed each of five phenotypes after a $30 \mathrm{~min}$ exposure to $0.5 \mathrm{~mm}$ muscimol $(\boldsymbol{A}, \boldsymbol{B})$ or $10 \mathrm{mg} / \mathrm{ml} \gamma$-hydroxybutyrate, GHB (C). Early L4 worms are designated by black bars $(n=25-30)$ and $L 4$ lethargus (leth) worms are designated by gray bars $(n=18-29)$; wt, Wild type. Severity of response is increased from 0 (no response) to 5 (complete paralysis). See Materials and Methods for full description of phenotypes. $D, G H B$ acts via the $\mathrm{GABA}_{\mathrm{A}}$ receptor UNC-49. Control worms ( $n=$ 8 -11) exhibited a dose-dependent inhibition of feeding when incubated on GHB (ANOVA, $p<0.00001)$, but unc-49(e382) worms $(n=11-14)$, lacking a functional $G_{A B A_{A}}$ receptor, did not decrease feeding (ANOVA, $p=0.082$ ).

motion are not only via the $u n c-49$ locus, but that other mechanisms are involved. During L4 lethargus, unc-49 mutants, like wild-type worms, were resistant to the paralyzing effects of muscimol (Fig. $4 B$ ).

In addition to muscimol, we found wild-type worms to be partially resistant to the GABAergic drug sodium oxybate (also known as $\gamma$-hydroxybutyrate or GHB) (Fig. $4 B$ ). We found that $\mathrm{GHB}$, an oxidative derivative of GABA (Pardi and Black, 2006), causes similar behavioral effects in worms as does muscimol (Fig. 4C) and, like muscimol (McIntire et al., 1993), appears to act partially via the gene $u n c-49$ (Fig. $4 D$ ), which encodes a $\mathrm{GABA}_{\mathrm{A}}$ receptor subunit.
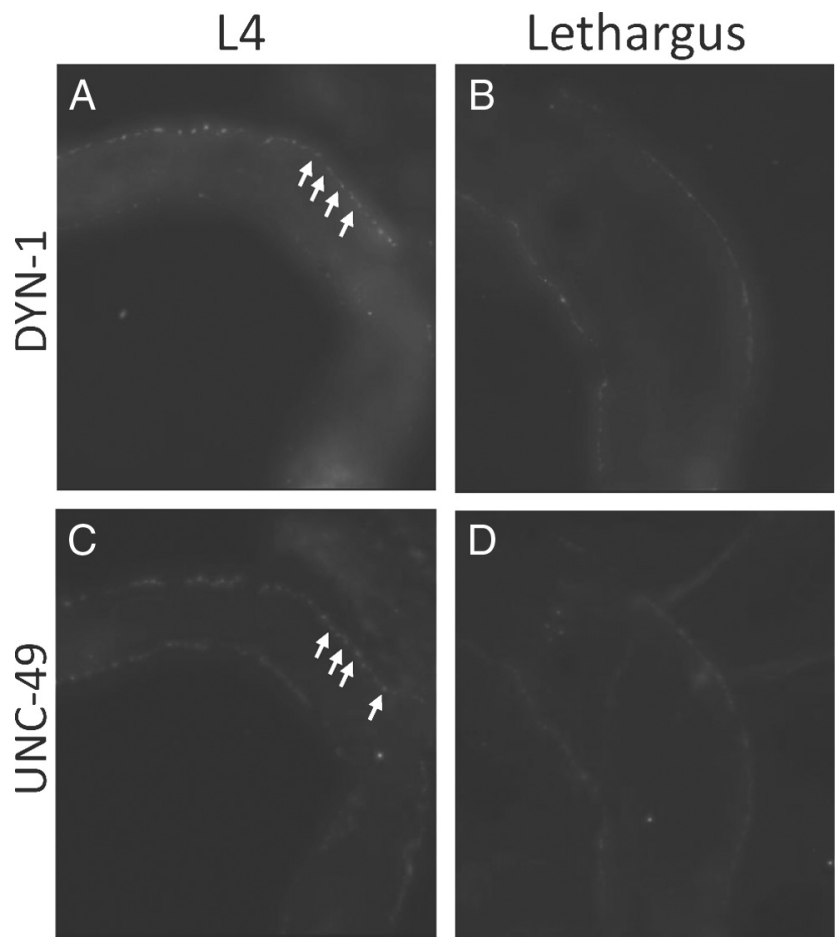

Figure 5. UNC-49 immunostaining shows no reduction during $L 4$ lethargus. DYN-1 and UNC-49 immunostaining of a mid-L4 worm $(\boldsymbol{A}, \boldsymbol{C})$ and of $L 4$ lethargus worms $(\boldsymbol{B}, \boldsymbol{D})$. Arrows point to examples of synaptic puncta.

\section{Expression of the GABA-A receptor gene unc- 49 is altered during lethargus}

The observation that L4 lethargus worms are partially resistant to $\mathrm{GABA}_{\mathrm{A}}$ receptor agonists suggests either that the total amount of $\mathrm{GABA}_{\mathrm{A}}$ receptor is reduced or that the function of this receptor is decreased. The overall protein levels of receptor in the membrane reflect a balance between new receptor synthesis and insertion and receptor degradation. The functional state of the receptor reflects its subunit composition (Bamber et al., 1999) as well as the $\mathrm{Cl}^{-}$potential across the membrane ( $\mathrm{Li}$ and $\mathrm{Xu}, 2008$ ).

In C. elegans, there is only one gene, called unc-49, known to encode $\mathrm{GABA}_{\mathrm{A}}$ receptor subunits. unc-49 is alternatively spliced to generate distinct receptor subunits (Bamber et al., 1999). To assess for the level of UNC-49 protein at the NMJ, we stained worms with an anti-UNC-49 antibody. To control for wormto-worm and stage-specific variability in staining, we performed a coimmunostaining experiment using the antibody DYN-1, which stains the presynaptic protein DYNAMIN (Hadwiger et al., 2010). Since the DYN-1 antibody staining did not change during lethargus (Fig. $5 A, B$, quantification not shown), we normalized our measurements of anti-UNC-49 staining to those of DYN-1 staining (see Materials and Methods). The normalized peak intensity of synaptic anti-UNC-49 fluorescence during L4 lethargus $(2.03 \pm 0.51)$ was not significantly different from the normalized peak intensity during the mid L4 stage $(3.14 \pm 1.71)$.

We considered the possibility that a change in the composition of the $\mathrm{GABA}_{\mathrm{A}}$ receptor modulates its function and perhaps its protein expression during lethargus. The function of the mammalian $\mathrm{GABA}_{\mathrm{A}}$ receptor is sensitive to the subunit composition (Simeone et al., 2003). One mechanism for regulating receptor subunit composition is via the transcriptional regulation 
Table 2. Increased expression of the UNC-49A splice isoform during L4 lethargus

\begin{tabular}{lllc}
\hline UNC-49 isoform & Relative expression (L4 lethargus/L4) & SD & $p$ value \\
\hline A & 1.92 & 0.159 & $<0.01$ \\
B & 0.97 & 0.05 & 0.998 \\
C (long + short) & 0.99 & 0.189 & 0.95 \\
\hline
\end{tabular}

of the individual subunits. The C. elegans unc-49 gene is alternatively spliced to generate four main isoforms of the GABA receptor UNC-49: UNC-49A, UNC-49B, UNC-49C (long), and UNC-49C (short). In mixed stages of animals, UNC-49A expression is much lower than that of the other subunits, and its functional role is unknown (Bamber et al., 1999). UNC-49B is essential for receptor function and can form a homomeric receptor (Bamber et al., 1999). When coexpressed with UNC-49B in a heterologous system, UNC-49C confers reduced GABA sensitivity and reduced $\mathrm{Cl}^{-}$conductance in comparison to the UNC-49B homomer alone (Bamber et al., 1999).

To evaluate for possible transcriptional changes during lethargus, we used quantitative reverse transcriptase PCR to compare GABA receptor transcripts levels between L4 and L4 lethargus worms (Table 2). Whereas UNC-49C and UNC-49B mRNA levels were unchanged during lethargus, UNC-49A levels were twofold greater during the L4 lethargus stage than during the $L 4$ stage. This finding suggests that the $\mathrm{GABA}_{\mathrm{A}}$ receptor subunit composition may differ between lethargus and other stages. This difference may affect receptor abundance and/or function.

\section{Discussion}

Starting with the observation that during lethargus worms are hypersensitive to aldicarb, we demonstrate that neuromuscular synaptic transmission is dynamic during development and that GABAergic transmission is reduced.

An initial concern in these experiments is that aldicarb hypersensitivity is a reflection of increased permeability of the exoskeleton to drugs during lethargus. Several lines of evidence argue that this is not the case: (1) We observed partial resistance and not hypersensitivity to muscimol and $\mathrm{GHB}$, indicating that worms are not generally hypersensitive to drugs during lethargus. (2) We did not observe uptake of Hoechst dye during L4 lethargus. (3) unc-25 mutants are hypersensitive to aldicarb during the adult stage but not during the lethargus stage. This lack of hypersensitivity during lethargus is not explained by a ceiling effect of the time course to paralysis because goa-1 mutants, who like unc-25 mutants are aldicarb-hypersensitive during the adult stage, are hypersensitive also during the lethargus stage. (4) We observed defects in GABAergic synaptic transmission using light, a nonpharmacological stimulus, to activate motor neurons. Thus, alteration in cuticle permeability does not explain aldicarb hypersensitivity during lethargus. Rather, the aldicarb assay results can be partially explained by an alteration in the balance between excitatory cholinergic and inhibitory GABAergic neuromuscular transmission during lethargus (Fig. $1 A$ ). GABAergic transmission is weakened.

The observed reduction in GABAergic synaptic transmission cannot fully explain the severe aldicarb hypersensitivity during lethargus relative to nonlethargus stages. If reduced GABA synaptic transmission during lethargus were the full explanation, one would expect the aldicarb sensitivity during lethargus of wild-type worms to parallel aldicarb sensitivity during the adult stage of $u n c-25$ mutants. Yet, wild-type aldi-
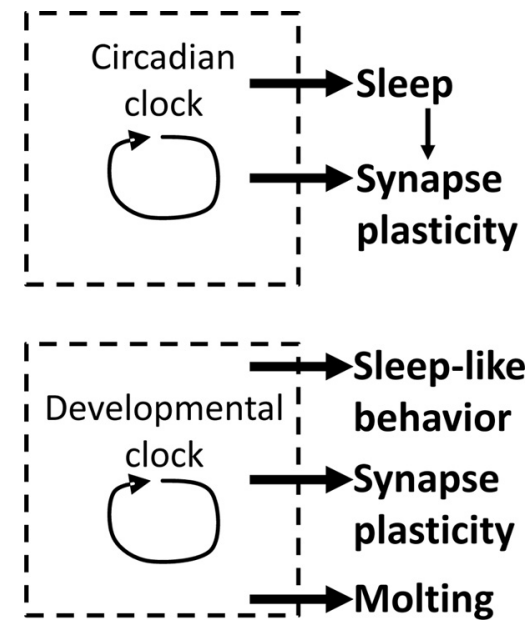

Figure 6. Parallels between the output of the circadian clock in vertebrates and Drosophila and the developmental clock in C. elegans. In vertebrates and in Drosophila, a circadian clock regulates the timing of sleep and also regulates the timing of synapse plasticity. At some synapses, the behavioral state of the animal also affects the synaptic plasticity. In C. elegans, a developmental clock controls three parallel processes: sleep-like behavior, synaptic plasticity, and molting.

carb sensitivity during lethargus is more severe than that of unc-25 adult worms (Fig. $3 B$ ). Therefore, there are additional factors contributing to the observed hypersensitivity during lethargus. There are a few possibilities that we have not yet explored. One possibility is that the accumulation of acetylcholine caused by aldicarb is affecting synapses other than those at the neuromuscular junction. For example, acetylcholine could be activating a neuron that promotes quiescence of locomotion. It is noteworthy that the ALA neuron, which can promote behavioral quiescence when activated by EGF ligands and is required for normal lethargus quiescence (Van Buskirk and Sternberg, 2007), expresses deg-3, which encodes a nicotinic acetylcholine receptor subunit (Treinin and Chalfie, 1995). Although we did not observe aldicarb resistance during lethargus in ceh-17 mutants, in which the quiescence-promoting effects of ALA activation by EGF are defective, ALA may be activated by non-EGF (i.e., by acetylcholine) means in this mutant. Furthermore, there might be heretofore undiscovered quiescence promoting cells that are activated by acetylcholine.

Another possible explanation for aldicarb hypersensitivity, and thus earlier paralysis, is impaired attachment of the muscle to the overlying hypodermis during lethargus. The attachment of the muscle to the hypodermis is via integrin-based hemidesmosomes, which show evidence of reorganization during lethargus (Zaidel-Bar et al., 2010). Sustained contraction may weaken this connection and result in irreversible paralysis. Mutants that impair muscle to hypodermis attachment display progressive paralysis (Plenefisch et al., 2000). However, unlike muscle attachment mutants, who show irreversible paralysis, the paralysis of lethargus worms on aldicarb is reversible when removed from the drug (data not shown).

Therefore, accelerated paralysis on aldicarb during lethargus is explained by reduced GABAergic synaptic transmission and by other unidentified mechanisms.

We observed a reduced behavioral response to GABAergic agonists during lethargus, and we detected a change in mRNA encoding for subunits of the $\mathrm{GABA}_{\mathrm{A}}$ receptor UNC-49. However, we did not detect a reduction in immunostaining for the UNC-49 
synaptic protein. In contrast, the aldicarb sensitivity curve for unc-25 mutants during lethargus and the optogenetic stimulation experiments suggest a near complete absence of synaptic transmission from GABAergic neurons during lethargus. Therefore, it is plausible that there is a reduction in GABAergic motor neuron presynaptic function, in addition to the reduction in postsynaptic function.

\section{Relationship of synaptic changes to developmental timing}

While lethargus has sleep-like behavioral properties (Raizen et al., 2008), we show by using multiple lines of evidence that the synaptic changes we observe during lethargus are not explained by the behavioral state of the animal. Rather, the synaptic plasticity is a function of the larval developmental stage. As such, this plasticity is related to other cyclical events with a periodicity tied to the molting cycle. The nematode molting cycle has similarities to circadian cycles in arthropods and mammals.

The gene lin-42, which encodes a protein that is the closest $C$. elegans relative to the circadian protein PERIOD, cycles with the larval molting cycle (Jeon et al., 1999). The cycling of lin-42 provides a molecular analog between the regulation of circadian timing and the regulation of larval developmental timing. Several genes encoding nuclear hormone receptors (NHRs) show cyclical expression during C. elegans larval development (Gissendanner et al., 2004). Cycling of NHR genes is a prominent feature of the circadian transcriptome in mammals (Yang et al., 2006) and functions both to modulate clock timing and to act as a link between the circadian clock and metabolism (Green et al., 2008). In addition to these molecular similarities between the C. elegans molting cycle and the mammalian circadian cycle, there are behavioral similarities as well, as lethargus has sleep-like properties (Raizen et al., 2008).

Therefore, the synaptic plasticity during the larval molting cycle in C. elegans can be compared to the synaptic plasticity observed during the adult circadian cycle in other animals. In mammals, Drosophila, and zebrafish, synaptic changes have been observed across circadian times. While circadian timing affects the plasticity in each of these species, the additional input of behavioral state (sleep vs wake) to this plasticity varies between species and between the specific synapse studied. In zebrafish, plasticity of hypocretin neuron synapses is primarily regulated by circadian factors and to a much lesser extent by behavioral state (Appelbaum et al., 2010). By contrast, in whole brains of fruit flies, levels of synaptic proteins are regulated primarily by behavioral state and not by time of day (Gilestro et al., 2009). Interestingly, in fish and Drosophila as well as in rodents (Donlea et al., 2009; Gilestro et al., 2009; Appelbaum et al., 2010; Liu et al., 2010a), synaptic down-scaling is observed at a circadian time that corresponds to sleep time.

Our observations suggest that a reduction in synaptic strength may be a conserved feature of the output of the clock, whether a circadian clock or a developmental clock (Fig. 6). The same metabolic milieu that is conducive to sleep-like behavior may also be conducive to synaptic downscaling. According to this view, synaptic downscaling is a byproduct of the metabolic state of the cell and organism but is not serving a specific purpose. An alternative view is that synaptic plasticity is serving a specific role during nervous system development and adult plasticity.

What function can weakening of synapses serve during $C$. elegans larval development? Newly hatched worms have 222 neurons. An additional 80 neurons are born postembryonically (Sul- ston and Horvitz, 1977). Most of these are born in the first larval stage and at least half of these neurons are motor neurons. The biological challenge posed by this nervous system growth is that over one-fourth of adult neurons must form new synapses and incorporate into the circuitry during larval development. But to form these new synapses, rearrangement of existing embryonic synapses may first need to occur. An example of such rearrangement is shown in the case of GABAergic motor neurons, called D neurons. The animal is born with six DD motor neurons, which initially form synapses on the ventral side of the animal. An additional 13 GABAergic motor neurons, termed the VD neurons, are born during the L1 stage. Like the DD neurons, VD neurons form synapses on ventral muscle cells. The preexisting ventral synapses made by DD neurons are eliminated and new DD synapses form on the dorsal muscles (White et al., 1978). Strikingly, DD neuron dorsal synaptogenesis occurs at the end of the L1 stage, coincident with the time of L1 lethargus (White et al., 1978; Hallam and Jin, 1998), and the elimination of the ventral DD synapses begins during L1 lethargus (Park et al., 2011). Therefore, one possible function served by the weakening of GABAergic synapses is to progress to elimination of these synapses.

Another GABAergic synapse that shows plasticity in lethargus is the synapse between the AVL and DVB motoneurons and enteric muscles in males. In L4 males, GABA released by these neurons causes contraction of the sphincter muscle and contributes to the expulsion step of the defecation cycle. In adult males, activation of this synapse leads to relaxation of the sphincter muscle (Reiner and Thomas, 1995). In the case of AVL/DVB synaptic plasticity, the change is postsynaptic, since the same neurotransmitter elicits an opposite behavioral response in L4 and adult. Therefore, weakening of synapses, and specifically GABAergic synapses, may serve a function more than once during lethargus periods.

\section{References}

Appelbaum L, Wang G, Yokogawa T, Skariah GM, Smith SJ, Mourrain P, Mignot E (2010) Circadian and homeostatic regulation of structural synaptic plasticity in hypocretin neurons. Neuron 68:87-98.

Bamber BA, Beg AA, Twyman RE, Jorgensen EM (1999) The Caenorhabditis elegans unc-49 locus encodes multiple subunits of a heteromultimeric GABA receptor. J Neurosci 19:5348-5359.

Bland JM, Altman DG (2004) The logrank test. BMJ 328:1073.

Boulin T, Gielen M, Richmond JE, Williams DC, Paoletti P, Bessereau JL (2008) Eight genes are required for functional reconstitution of the Caenorhabditis elegans levamisole-sensitive acetylcholine receptor. Proc Natl Acad Sci U S A 105:18590-18595.

Brenner S (1974) The genetics of Caenorhabditis elegans. Genetics 77:71-94. Chase DL, Koelle MR (2007) Biogenic amine neurotransmitters in C. elegans. WormBook 20:1-15.

Daniels SA, Ailion M, Thomas JH, Sengupta P (2000) egl-4 acts through a transforming growth factor-beta/SMAD pathway in Caenorhabditis elegans to regulate multiple neuronal circuits in response to sensory cues. Genetics 156:123-141.

Davis MW, Somerville D, Lee RY, Lockery S, Avery L, Fambrough DM (1995) Mutations in the Caenorhabditis elegans Na,K-ATPase $\alpha$-subunit gene, eat-6, disrupt excitable cell function. J Neurosci 15:8408-8418.

de la Cruz IP, Levin JZ, Cummins C, Anderson P, Horvitz HR (2003) sup-9, sup-10, and unc-93 may encode components of a two-pore $\mathrm{K}^{+}$channel that coordinates muscle contraction in Caenorhabditis elegans. J Neurosci 23:9133-9145.

Donlea JM, Ramanan N, Shaw PJ (2009) Use-dependent plasticity in clock neurons regulates sleep need in Drosophila. Science 324:105-108. 
Fernández MP, Berni J, Ceriani MF (2008) Circadian remodeling of neuronal circuits involved in rhythmic behavior. PLoS Biol 6:e69.

Frand AR, Russel S, Ruvkun G (2005) Functional genomic analysis of $C$. elegans molting. PloS Biol 3:e312.

Gendrel M, Rapti G, Richmond JE, Bessereau JL (2009) A secreted complement-control-related protein ensures acetylcholine receptor clustering. Nature 461:992-996.

Gilestro GF, Tononi G, Cirelli C (2009) Widespread changes in synaptic markers as a function of sleep and wakefulness in Drosophila. Science 324:109-112.

Gissendanner CR, Sluder AE (2000) nhr-25, the Caenorhabditis elegans ortholog of $f t z-f 1$, is required for epidermal and somatic gonad development. Dev Biol 221:259-272.

Gissendanner CR, Crossgrove K, Kraus KA, Maina CV, Sluder AE (2004) Expression and function of conserved nuclear receptor genes in Caenorhabditis elegans. Dev Biol 266:399-416.

Green CB, Takahashi JS, Bass J (2008) The meter of metabolism. Cell 134:728-742.

Hadwiger G, Dour S, Arur S, Fox P, Nonet ML (2010) A monoclonal antibody toolkit for C. elegans. PLoS One 5:e10161.

Hallam SJ, Jin Y (1998) Lin-14 regulates the timing of synaptic remodelling in Caenorhabditis elegans. Nature 395:78-82.

Hegstrom CD, Truman JW (1996) Synapse loss and axon retraction in response to local muscle degeneration. J Neurobiol 31:175-188.

Hoogewijs D, Houthoofd K, Matthijssens F, Vandesompele J, Vanfleteren JR (2008) Selection and validation of a set of reliable reference genes for quantitative sod gene expression analysis in C. elegans. BMC Mol Biol 9:9.

Jeon M, Gardner HF, Miller EA, Deshler J, Rougvie AE (1999) Similarity of the C. elegans developmental timing protein LIN-42 to circadian rhythm proteins. Science 286:1141-1146.

Jin Y, Jorgensen E, Hartwieg E, Horvitz HR (1999) The Caenorhabditis elegans gene unc- 25 encodes glutamic acid decarboxylase and is required for synaptic transmission but not synaptic development. J Neurosci 19:539-548.

Kamath RS, Fraser AG, Dong Y, Poulin G, Durbin R, Gotta M, Kanapin A, Le Bot N, Moreno S, Sohrmann M, Welchman DP, Zipperlen P, Ahringer J (2003) Systematic functional analysis of the Caenorhabditis elegans genome using RNAi. Nature 421:231-237.

Kim K, Li C (2004) Expression and regulation of an FMRFamide-related neuropeptide gene family in Caenorhabditis elegans. J Comp Neurol 475:540-550.

Kostrouchova M, Krause M, Kostrouch Z, Rall JE (2001) Nuclear hormone receptor $\mathrm{CHR} 3$ is a critical regulator of all four larval molts of the nematode Caenorhabditis elegans. Proc Natl Acad Sci U S A 98:7360-7365.

Leung B, Hermann GJ, Priess JR (1999) Organogenesis of the Caenorhabditis elegans intestine. Dev Biol 216:114-134.

Levine RB, Truman JW (1982) Metamorphosis of the insect nervous system: changes in morphology and synaptic interactions of identified neurones. Nature 299:250-252.

LiK, Xu E (2008) The role and the mechanism of gamma-aminobutyric acid during central nervous system development. Neurosci Bull 24:195-200.

Liewald JF, Brauner M, Stephens GJ, Bouhours M, Schultheis C, Zhen M, Gottschalk A (2008) Optogenetic analysis of synaptic function. Nat Methods 5:895-902.

Liu Q, Hollopeter G, Jorgensen EM (2009) Graded synaptic transmission at the Caenorhabditis elegans neuromuscular junction. Proc Natl Acad Sci U S A 106:10823-10828.

Liu Z, Chen Y, Wang D, Wang S, Zhang YQ (2010b) Distinct presynaptic and postsynaptic dismantling processes of Drosophila neuromuscular junctions during metamorphosis. J Neurosci 30:11624-11634.

Liu ZW, Faraguna U, Cirelli C, Tononi G, Gao XB (2010a) Direct evidence for wake-related increases and sleep-related decreases in synaptic strength in rodent cortex. J Neurosci 30:8671-8675.

Livak KJ, Schmittgen TD (2001) Analysis of relative gene expression data using real-time quantitative PCR and the 2(-Delta Delta C(T)) method. Methods 25:402-408.

Locke CJ, Kautu BB, Berry KP, Lee SK, Caldwell KA, Caldwell GA (2009) Pharmacogenetic analysis reveals a post-developmental role for Rac GTPases in Caenorhabditis elegans GABAergic neurotransmission. Genetics 183:1357-1372.

Mahoney TR, Luo S, Nonet ML (2006) Analysis of synaptic transmission in
Caenorhabditis elegans using an aldicarb-sensitivity assay. Nat Protoc 1:1772-1777.

McIntire SL, Jorgensen E, Horvitz HR (1993) Genes required for GABA function in Caenorhabditis elegans. Nature 364:334-337.

Mehnert KI, Beramendi A, Elghazali F, Negro P, Kyriacou CP, Cantera R (2007) Circadian changes in Drosophila motor terminals. Dev Neurobiol 67:415-421.

Meli VS, Osuna B, Ruvkun G, Frand AR (2010) MLT-10 defines a family of DUF644 and proline-rich repeat proteins involved in the molting cycle of Caenorhabditis elegans. Mol Biol Cell 21:1648-1661.

Miller KG, Emerson MD, Rand JB (1999a) Goalpha and diacylglycerol kinase negatively regulate the Gqalpha pathway in C. elegans. Neuron 24:323-333.

Miller KG, Emerson MD, Rand JB (1999b) Goalpha and diacylglycerol kinase negatively regulate the Gqalpha pathway in C. elegans. [see comment]. Neuron 24:323-333.

Moribe H, Yochem J, Yamada H, Tabuse Y, Fujimoto T, Mekada E (2004) Tetraspanin protein (TSP-15) is required for epidermal integrity in Caenorhabditis elegans. J Cell Sci 117:5209-5220.

Nagel G, Brauner M, Liewald JF, Adeishvili N, Bamberg E, Gottschalk A (2005) Light activation of channelrhodopsin-2 in excitable cells of Caenorhabditis elegans triggers rapid behavioral responses. Curr Biol 15:2279-2284.

Nakanishi N, Yuan D, Jacobs DK, Hartenstein V (2008) Early development, pattern, and reorganization of the planula nervous system in Aurelia (Cnidaria, Scyphozoa). Dev Genes Evol 218:511-524.

Nonet ML, Staunton JE, Kilgard MP, Fergestad T, Hartwieg E, Horvitz HR, Jorgensen EM, Meyer BJ (1997) Caenorhabditis elegans rab-3 mutant synapses exhibit impaired function and are partially depleted of vesicles. J Neurosci 17:8061-8073.

Pardi D, Black J (2006) gamma-Hydroxybutyrate/sodium oxybate: neurobiology, and impact on sleep and wakefulness. CNS Drugs 20:993-1018.

Park M, Watanabe S, Poon VY, Ou CY, Jorgensen EM, Shen K (2011) CYY1/Cyclin Y and CDK-5 differentially regulate synapse elimination and formation for rewiring neural circuits. Neuron 70:742-757.

Partridge FA, Tearle AW, Gravato-Nobre MJ, Schafer WR, Hodgkin J (2008) The C. elegans glycosyltransferase BUS- 8 has two distinct and essential roles in epidermal morphogenesis. Dev Biol 317:549-559.

Plenefisch JD, Zhu X, Hedgecock EM (2000) Fragile skeletal muscle attachments in dystrophic mutants of Caenorhabditis elegans: isolation and characterization of the mua genes. Development 127:1197-1207.

Raizen DM, Zimmerman JE, Maycock MH, Ta UD, You YJ, Sundaram MV, Pack AI (2008) Lethargus is a C. elegans sleep like state. Nature 451:569-572.

Reiner DJ, Thomas JH (1995) Reversal of a muscle response to GABA during C. elegans male development. J Neurosci 15:6094-6102.

Richmond J (2005) Synaptic function. WormBook 30:1-14.

Robatzek M, Niacaris T, Steger K, Avery L, Thomas JH (2001) eat-11 encodes GPB-2, a Gbeta(5) ortholog that interacts with G(o)alpha and G(q)alpha to regulate C. elegans behavior. Curr Biol 11:288-293.

Rocha L, Ackermann RF, Engel J Jr (1996) Chronic and single administration of pentylenetetrazol modifies benzodiazepine receptor-binding: an autoradiographic study. Epilepsy Res 24:65-72.

Seifert M, Schmidt E, Baumeister R (2006) The genetics of synapse formation and function in Caenorhabditis elegans. Cell Tissue Res 326:273-285.

Simeone TA, Donevan SD, Rho JM. Molecular biology and ontogeny of gamma-aminobutyric acid (GABA) receptors in the mammalian central nervous system. J Child Neurol 18:39-49.

Stiernagle T (2006) Maintenance of C. elegans. WormBook 11:1-11.

Sulston JE, Horvitz HR (1977) Post-embryonic cell lineages of the nematode, Caenorhabditis elegans. Dev Biol 56:110-156.

Timmons L, Fire A (1998) Specific interference by ingested dsRNA. Nature 395:854.

Tissot M, Stocker RF (2000) Metamorphosis in Drosophila and other insects: the fate of neurons throughout the stages. Prog Neurobiol 62:89-111.

Treinin M, Chalfie M (1995) A mutated acetylcholine receptor subunit causes neuronal degeneration in C. elegans. Neuron 14:871-877.

Van Buskirk C, Sternberg PW (2007) Epidermal growth factor signaling induces behavioral quiescence in Caenorhabditis elegans. Nat Neurosci 10:1300-1307.

Van Buskirk C, Sternberg PW (2010) Paired and LIM class homeodomain 
proteins coordinate differentiation of the C. elegans ALA neuron. Development 137:2065-2074.

Vashlishan AB, Madison JM, Dybbs M, Bai J, Sieburth D, Ch'ng Q, Tavazoie M, Kaplan JM (2008) An RNAi screen identifies genes that regulate GABA synapses. Neuron 58:346-361.

Wang ZW, Saifee O, Nonet ML, Salkoff L (2001) SLO-1 potassium channels control quantal content of neurotransmitter release at the C. elegans neuromuscular junction. Neuron 32:867-881.

Watanabe H, Fujisawa T, Holstein TW (2009) Cnidarians and the evolutionary origin of the nervous system. Dev Growth Differ 51:167-183.

White JG, Albertson DG, Anness MA (1978) Connectivity changes in a class of motoneurone during the development of a nematode. Nature $271: 764-766$
Williams SN, Locke CJ, Braden AL, Caldwell KA, Caldwell GA (2004) Epileptic-like convulsions associated with LIS-1 in the cytoskeletal control of neurotransmitter signaling in Caenorhabditis elegans. Hum Mol Genet 13:2043-2059.

Yang X, Downes M, Yu RT, Bookout AL, He W, Straume M, Mangelsdorf DJ, Evans RM (2006) Nuclear receptor expression links the circadian clock to metabolism. Cell 126:801-810.

You YJ, Kim J, Raizen DM, Avery L (2008) Insulin, cGMP, and TGF-beta signals regulate food intake and quiescence in $C$. elegans: a model for satiety. Cell Metab 7:249-257.

Zaidel-Bar R, Miller S, Kaminsky R, Broday L (2010) Molting-specific downregulation of $C$. elegans body-wall muscle attachment sites: the role of RNF-5 E3 ligase. Biochem Biophys Res Commun 395:509-514. 